\title{
Functional Modelling in Evolvable Assembly Systems
}

\author{
David Sanderson ${ }^{10000-0002-8675-4991]}$ and Jack C Chaplin ${ }^{10000-0003-3282-2386]}$ and Svetan \\ Ratchev 1 [0000-0001-9955-2806] \\ ${ }^{1}$ Institute for Advanced Manufacturing, Advanced Manufacturing Building, Jubilee Campus, \\ University of Nottingham, UK, NG8 1BB \\ (firstname. lastname) @nottingham.ac.uk
}

\begin{abstract}
The design and reconfiguration of adaptive production systems is a key driver in modern advanced manufacturing. We summarise the use of an approach from the field of functional modelling to capture the function, behaviour, and structure of a system. This model is an integral part of the Evolvable Assembly Systems architecture, allowing the system to adapt its behaviour in response to changing product requirements. The integrated approach is illustrated with an example taken from a real EAS instantiation.
\end{abstract}

Keywords: Architecture, Evolvable Assembly Systems, Functional Modelling, Multi-Agent Systems.

\section{$1 \quad$ Introduction}

The manufacturing industry as a whole is facing increased market unpredictability and labour costs, as well as growing consumer demand for highly personalised goods and services with a shorter time to market and increased product diversity [1]. In order to incorporate these changes, manufacturing systems have begun to take advantage of adaptive control for flexibility, resilience, and monitoring. Manufacturing companies in many sectors are therefore investigating smart, flexible, and adaptive manufacturing lines that can autonomously self-heal, self-adapt, and reconfigure in response to changing product requirements. This is typified by the 'batch-size-of-one' problem, wherein each product may be unique and the manufacturing system must be capable of carrying out different production processes as required by the current product.

A common approach to these problems is that of cyber-physical systems [2], often implemented as a multi-agent system [3]. One such implementation is that of the Evolvable Assembly Systems project [4], which combines a behavioural framework based on functional modelling with a multi-agent cyber-physical systems architecture.

\section{$2 \quad$ Functional Modelling}

The modelling, design, and integration of assembly systems is primarily based on process requirements. This requires that the capabilities of a production resource are captured and can be reasoned about. There are two main ways of capturing the capabilities 
of a production resource: by considering the processes that the resource can perform, and by considering how their structure and behaviour relate to their intended functions. We take the second approach, based on the Function-Behaviour-Structure (FBS) formalisations by Gero, Rosenman, Umeda, and others in the field of functional modelling [5-11]. Consequently, we define Function as an abstraction of behaviour for a specific use or purpose, Behaviour as state transitions from input to output, and Structure as the physical model of the system and subsystem, and the connections between them.

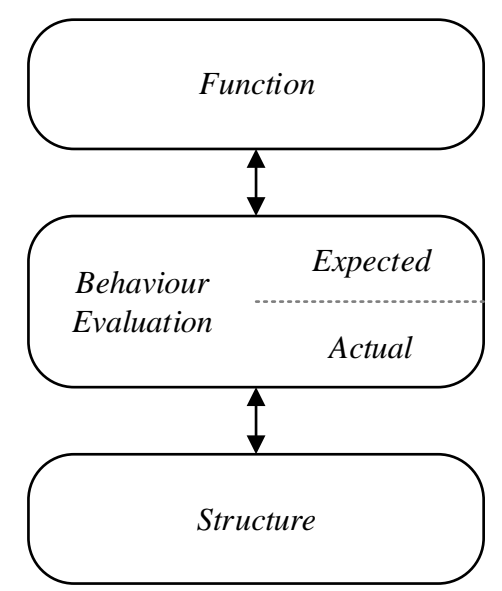

Fig. 1. Overview of the functional modelling behavioural approach

The behavioural framework shown in Fig. 1 is at the core of the Evolvable Assembly Systems approach. The required set of functions is used to determine the behaviours that are expected to fulfil those functions. The structure of the system is then designed, and the actual behaviour expressed by that structure is compared to the expected behaviour. The algorithmic basis for this process of distributed behavioural evaluation is described in more detail in [12].

Each resource in the system consists of some structure and corresponding behaviour(s). Some resources also have multiple configurations. These are managed by an intelligent agent; all agents in the system communicate with each other to provide distributed control based on a joint system model that provides both operational data, and the coordination for the distributed behavioural evaluation. 


\section{Evolvable Assembly Systems}

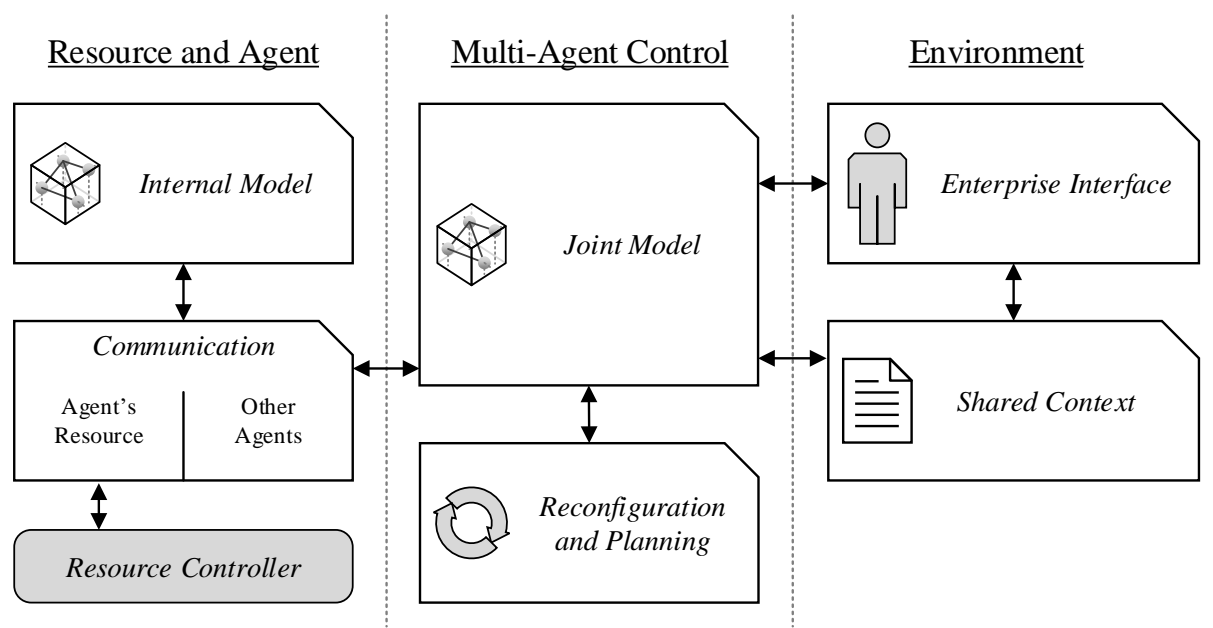

Fig. 2. An agent-oriented view of EAS architecture

An agent-oriented view of the EAS architecture is shown in Fig. 2. Each resource in the system is controlled at a high level by an intelligent agent - an encapsulated piece of software that makes control decisions based on available information. Evolvable Assembly Systems uses the Beliefs-Desires-Intentions agent paradigm [13], so this information is stored as beliefs, and translated into immediate intentions (plans) in order to accomplish long term desires (goals). The intentions of the agent are executed through behaviour in the system, based on the behaviours that can be expressed by the structure of the resource that is being controlled.

Each resource in the system can be defined as a set of structures and associated behaviours using Business to Manufacturing Markup Language (B2MML) based on the ISA-95 standard $[14,15]$. To enable an agent to control a resource, this description is used to generate an interface. This interface connects the agent with the PLC, controller, or similar that provides the low-level control for the resource hardware. This interface layer allows the agent core to remain the same whilst still providing control for a variety of hardware archetypes.

\section{Shared Context}

Although each agent is responsible for local control of the resource it is attached to, the collection of agents provide a distributed agent control layer for the whole production system. Communication between these agents is based on the ubiquitous sharing of contextual information.

This contextual information covers all aspects of the system. At the most fundamental level, it describes the product that is to be produced by the system, and the produc- 
tion capabilities of each resource in the system as well as any "joint capabilities" resulting from combinations of resources. This is further enhanced by information about the state of the product and system, for example the pose of a robot arm, the location of a pallet, or relevant metrology data. The context also provides a link between the EAS control system and the rest of the enterprise in which it is situated. This may include control systems for other assembly cells, or higher-level enterprise information systems. All of this information is stored in a context layer that is accessible to all agents as required. This shared context is implemented using a publish-subscribe data distribution service [16] and illustrated in Fig. 3.

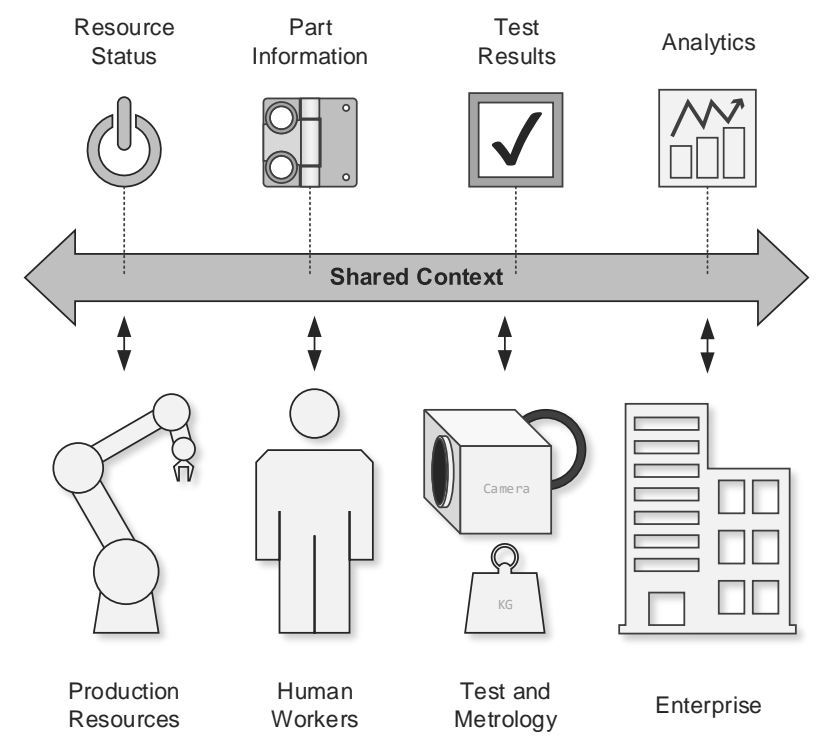

Fig. 3. Shared context data distribution

\section{Implementation Example}

This system has been implemented in a real precision assembly demonstrator (PAD) at the University of Nottingham, shown in Fig. 4. The demonstrator is designed to assemble interior hinges from the automotive industry. Each product is defined by a recipe file that indicates the détente force - achieved by the configuration of ball-spring pairs added to the hinge. Because each hinge produced by the system could be unique, these recipe files are a way to formalise the batch-size of one requirements in the system. 


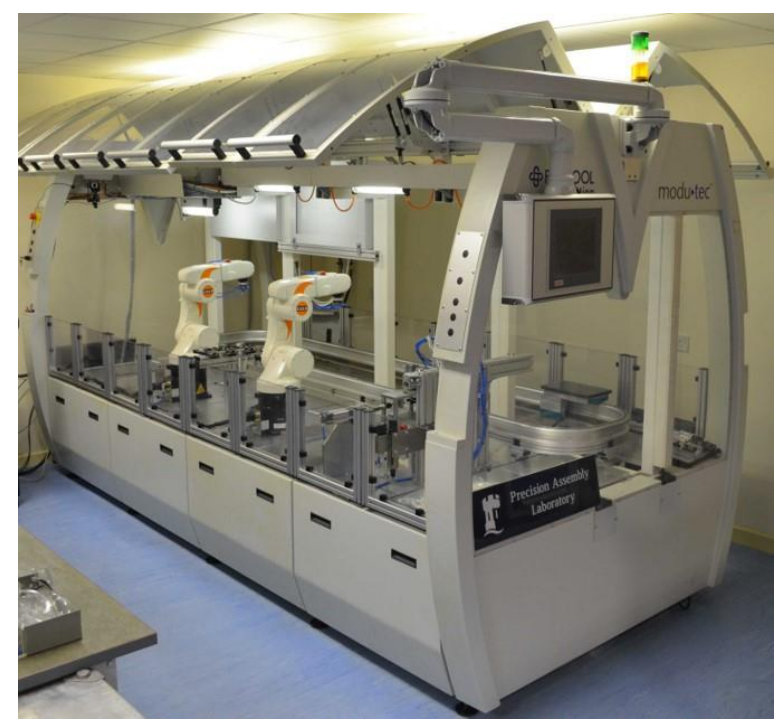

Fig. 4. The Precision Assembly Demonstrator (PAD)

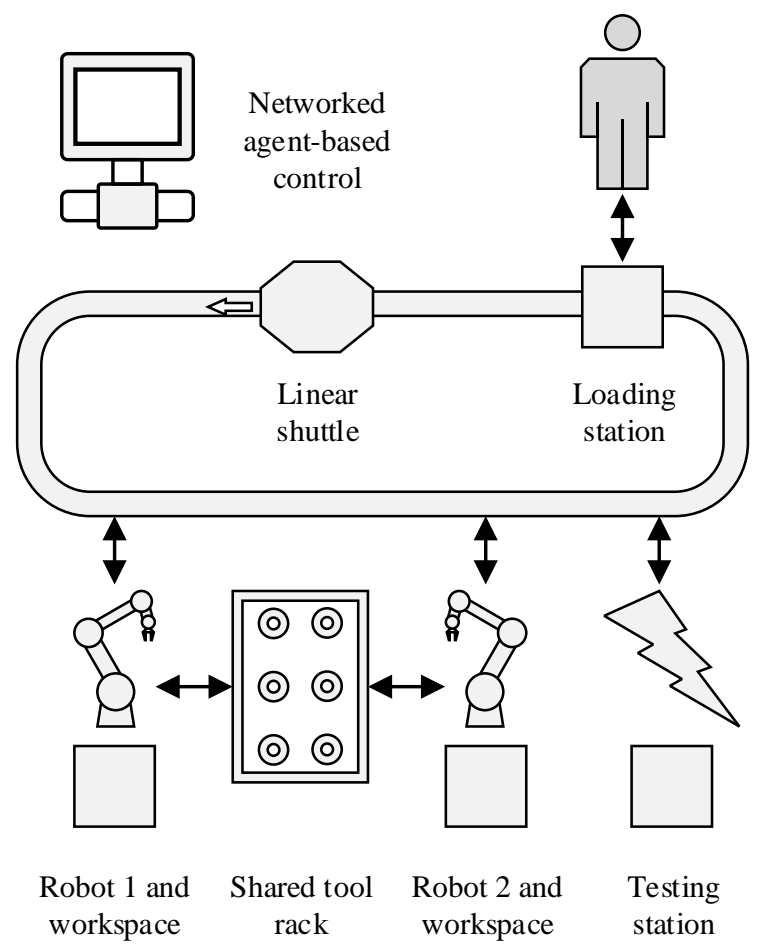

Fig. 5. Layout of the PAD 
The demonstrator is based on a Feintool Modutec assembly cell, and consists of two KUKA KR5 sixx R650 robots alongside a testing station with visual inspection and force testing equipment, connected via a linear shuttle system. Each robot has an individual working area, as does the testing station. The robots share a tool rack, giving them each access to a variety of different end effectors via an automatic tool changer. The whole system is accessible via a part loading station, where pallets of unassembled parts are loaded, and pallets with completed products are removed. A diagram of the demonstrator can be seen in Fig. 5 .

Table 1. Behaviours available to each station in the PAD

\begin{tabular}{lll}
\hline Station & Behaviour & Sub-behaviour (optional) \\
\hline \hline Shuttle & Move & \\
\hline Loading station & $\begin{array}{l}\text { Add pallet } \\
\text { Remove pallet }\end{array}$ & \\
\hline Robot 1 / 2 & Pick and place & To Conveyor \\
& & From Conveyor \\
& & Match leaves \\
& & Insert pin \\
& & Insert spring(s) \\
& & Insert ball(s) \\
& Change end effector & Gripper 1 \\
& & Gripper 2 \\
& & Gripper 3 \\
& & Spring gripper \\
& & Ball gripper \\
& & Press \\
\hline Testing station & Pick and place & To Conveyor \\
& Fision test & From Conveyor \\
& & (with force as variable) \\
\hline
\end{tabular}

In order to assemble the hinges, each subsystem has a number of behaviours, as shown in Table 1. The robots can change end effector and have the following pick and place behaviours: move the pallet between the shuttle system and the workspace; match the hinge leaves; insert the hinge pin; insert a variable number of springs; insert a variable number of balls; fit the retainer. The testing station has pick and place behaviours to move the product between the shuttle system and the workspace, as well as a force testing behaviour to test the détente force, and a vision testing behaviour to ensure the product is correctly assembled. The shuttle system has only one behaviour: moving from one station to another. The loading station and operator can only load parts into 
the system, and remove parts from the system. Note that for readability of Table $\mathbf{1}$ we have not shown the dependencies between end effector and pick and place behaviours. For example, the "spring gripper" end effector is required to fit a spring, but grippers 1-3 are interchangeable for some other tasks (moving the pallet, etc). More detail on the physical demonstration platform itself can be found in [17].

These behaviours are then adapted by the agent controlling the relevant resource in order to produce the product specified by the recipe file - that is, accomplish the required function inherent in the new product. Each agent may further be able to adapt some or all of the structure of its resource. In the case of our example, the shared tool rack provides a selection of end effectors. The selection of a new end effector changes both the structure and behaviour of the resource.

As the "loading station" behaviours are carried out by an operator they are logically part of the system, but do not have to be programmed into an agent - the operator only needs some way to tell the rest of the system what behaviour has been carried out.

\section{$6 \quad$ Summary and Acknowledgements}

This paper has described the agent-oriented architecture of Evolvable Assembly Systems in the context of the FBS approach from function modelling. This approach allows the system to accurately model the adaptive structure and behaviour of the system, and leverage them to achieve the system functions required by the changing product requirements of a batch-size of one scenario. This approach has been demonstrated on a real demonstration cell at the University of Nottingham.

The authors gratefully acknowledge the support provided by UK EPSRC Evolvable Assembly Systems (EP/K018205/1).

\section{References}

1. Kagermann, H., Helbig, J., Hellinger, A., Wahlster, W.: Recommendations for Implementing the Strategic Initiative INDUSTRIE 4.0: Securing the Future of German Manufacturing Industry; Final Report of the Industrie 4.0 Working Group. (2013).

2. Monostori, L.: Cyber-physical Production Systems: Roots, Expectations and R\&D Challenges. Procedia CIRP. 17, 9-13 (2014).

3. Wooldridge, M., Jennings, N.R.: Intelligent agents: Theory and practice. Knowl. Eng. Rev. 10, 115-152 (1995).

4. Chaplin, J.C., Bakker, O.J., de Silva, L., Sanderson, D., Kelly, E., Logan, B., Ratchev, S.M.: Evolvable Assembly Systems: A Distributed Architecture for Intelligent Manufacturing. IFAC-PapersOnLine. 48, 2065-2070 (2015).

5. Gero, J.S.: Design Prototypes: A Knowledge Representation Schema for Design. AI Mag. 11, 26-36 (1990).

6. Gero, J.S., Kannengiesser, U.: A function-behavior-structure ontology of processes. AI EDAM Artif. Intell. Eng. Des. Anal. Manuf. 21, 379-391 (2007).

7. Rosenman, M.., Gero, J..: Purpose and function in design: from the socio-cultural to the techno-physical. Des. Stud. 19, 161-186 (1998). 
8. Umeda, Y., Takeda, H., Tomiyama, T., Yoshikawa, H.: Function, behaviour, and structure. In: Applications of Artificial Intelligence in Engineering V. pp. 177-193 (1990).

9. Umeda, Y., Ishii, M., Yoshioka, M., Shimomura, Y., Tomiyama, T.: Supporting conceptual design based on the function-behavior-state modeler. Artif. Intell. Eng. Des. Anal. Manuf. 10, 275-288 (1996).

10. Sasajima, M., Kitamura, Y.: FBRL: A Function and Behavior Representation Language. In: Proceedings of the 14th International Joint Conferences on Artificial Intelligence (IJCAI). pp. 1830-1836 (1995).

11. Mizoguchi, R., Kitamura, Y.: Foundation of Knowledge Systematization: Role of Ontological Engineering. In: Industrial Knowledge Management. pp. 17-36. Springer London, London (2001).

12. de Silva, L., Felli, P., Chaplin, J.C., Logan, B., Sanderson, D., Ratchev, S.: Synthesising Industry-Standard Manufacturing Process Controllers. In: Proceedings of the 16th Conference on Autonomous Agents and MultiAgent Systems. pp. 1811-1813. International Foundation for Autonomous Agents and Multiagent Systems (2017).

13. Rao, A.S., Georgeff, M.P.: BDI agents: From theory to practice. In: Proceedings of the first international conference on multi-agent systems (ICMAS-95). pp. 312-319. San Francisco, CA (1995).

14. Business To Manufacturing Markup Language Operations Schedule Version 6.0, https://services.mesa.org/ResourceLibrary.

15. ANSI/ISA-95, Enterprise-Control System Integration, Parts 1-5, https://www.isa.org/standards-publications/.

16. Data Distribution Service, http://www.omg.org/spec/DDS/Current.

17. Antzoulatos, N., Castro, E., de Silva, L., Ratchev, S.: Interfacing Agents with an Industrial Assembly System for "Plug and Produce": (Demonstration). In: Proceedings of the 2015 International Conference on Autonomous Agents and Multiagent Systems. pp. 1957-1958. International Foundation for Autonomous Agents and Multiagent Systems, Richland, SC (2015). 